\title{
The burden of tuberculosis in Iran, A 12- year population-based study
}

\author{
Mohammad Khajedaluee ${ }^{1}$, Mahshid Nasehi², Saeid Sharafii ${ }^{3}$, Maliheh Dadgarmoghaddam*1(D) \\ Received: 29 Jan 2019 \\ Published: 25 Jan 2021
}

\begin{abstract}
Background: Tuberculosis (TB) is a major global health problem, so for better planning in the health sector, it is necessary to know the real burden of tuberculosis in our country. The main aim of this study was to calculate the burden of TB for the Iranian population in 2001-2012.

Methods: The Disability Adjusted Life Years (DALYs) index was calculated using a computer model (DisMod version II) in Iran between the years 2001 and 2012. DALYs are age-weighted $(\beta=0.04)$ and are discounted for time preference $(\mathrm{r}=0.03)$.

Results: The trend was decreasing from 2001 till 2006, and after it, there is an increasing trend. The incidence was more in younger age groups and in female, and the YLL is higher in men (11744 in male vs 7897 in female in 2012); it showed that the life lost is higher in men. The YLD in men and women are very close. In comparison, the DALY of TB was higher in men than women.

Conclusion: In the present investigation, it has been found that the overall tendency to get TB was higher in the female population. It shows that the incidence was higher in the younger age groups but the mortality was higher in the elderly groups. It that shows the significant success of the country in controlling the disease.
\end{abstract}

Keywords: Tuberculosis, Burden of disease, Disability-Adjusted life Years

Conflicts of Interest: None declared

Funding: None

*This work has been published under CC BY-NC-SA 1.0 license.

Copyright $₫$ Iran University of Medical Sciences

Cite this article as: Khajedaluee M, Nasehi M, Sharafii S, Dadgarmoghaddam M. The burden of tuberculosis in Iran, A 12- year population-based study. Med J Islam Repub Iran. 2021 (25 Jan);35:13. https://doi.org/10.47176/mjiri.35.13

\section{Introduction}

Tuberculosis (TB) is a major global health problem and remains an important etiological factor for mortality among infectious diseases. It ranks as a leading cause of death worldwide such as the human immunodeficiency virus (HIV). There were an estimated 9.6 million new TB cases in 2014 (5.4 million among men, 3.2 million among women, and 1.0 million among children) (1).

In 2014, 1.5 million TB deaths were reported (1.1 million among HIV-negative people and 0.4 million among HIVpositive people) (1). The number of TB deaths is unacceptably high, while it was stated that with a timely diagnosis and treatment, the disease could be cured.

Corresponding author: Dr Maliheh Dadgarmoghaddam, dadgarmm@mums.ac.ir

1. Department of Community Medicine, Faculty of Medicine, Mashhad University of medical Sciences, Mashhad, Iran

2. Department of Epidemiology, Faculty of Public Health, Iran University of Medical Sciences, Tehran, Iran

Center for control of communicable disease, Tehran, Iran
For the past decade, the main goal was the reduction of the TB burden based on the 2015 global targets of the Millennium Development Goals (MDGs). According to the targets, TB incidence should decrease (MDG Target 6.c) and that TB prevalence and mortality rates should be halved compared with their 1990 levels (2).

In Iran, important steps have been taken to control tuberculosis and has brought gains.

1. Reducing the reported incidence of tuberculosis from 140 to 14 per 100,000 people (over a period of fifty years)

2. A $34 \%$ reduction in the incidence of tuberculosis over the course of 23 years (from 32 per 1,000 population in

\section{$\uparrow$ What is "already known" in this topic:}

Important steps have been taken to control tuberculosis and have brought gains such as reducing the incidence, prevalence and death of tuberculosis.

\section{$\rightarrow$ What this article adds:}

In this study, it has been found that the overall tendency to get TB was higher in the female population. It shows that the incidence was higher in the younger age groups. 
1990 to 21 per 100,000 population in 2013)

3. A $36 \%$ reduction in the prevalence of TB in 23 years (from 50 cases per 100,000 population in 1990 to 32 per 100,000 population in 2013 )

4. A $36 \%$ reduction in tuberculosis deaths over a period of 23 years (from 5 per 100,000 population in 1990 to 3.2 per 100,000 population in 2013) (3).

Goals were also planned based on the horizon of the year 1404 as below:

1. Increasing the rate of case finding of smear positive pulmonary TB to more than $85 \%$

2. Increasing the treatment success rate of smear positive pulmonary TB in excess of $90 \%$

3. Decrease in tuberculosis outbreak by $30 \%$ compared to 2008

4. Decrease in tuberculosis mortality by $30 \%$ compared to 2008 (4).

The methods currently used by WHO to estimate the incidence of TB are grouped into four major categories:

1. Case notification data combined with an expert opinion about case detection gaps.

2. Results from national TB prevalence surveys.

3. Notifications in high-income countries adjusted by a standard factor to account for under-reporting and underdiagnosis.

4. Results from inventory/capture-recapture studies (1).

To understand the real burden of TB in our country, we need to calculate the burden according to the available data instead of any estimation. Therefore, we can plan better to move toward the MDGs. So the main aim of this study was to calculate the burden of TB for the Iranian population in 2001-2012.

\section{Methods}

This study investigates the burden of Tuberculosis according to data from the tuberculosis registration system in the years 2001 to 2012 .

For the calculation, we need indexes such as disease morbidity (incidence, prevalence, disease duration, remission rate) and mortality (disease-specific death rate, fatality rate, and relative death rate). Data on the incidence and outcome of treatment success, treatment failure and death were ex- tracted from the prevention and control of tuberculosis $\mathrm{Bu}-$ reau in Ministry of Health according to sex and age in the years 2001 to 2012 .

For calculating the burden of TB, we also need the demographic characteristics of the target population (age, gender, and general mortality rate).

Demographic information including age and gender and the crude death were extracted from the registry of the Department of Civil Registration. Census data and population estimates were gathered from the data of the health department of the Ministry of Health. The total population of the country was collected from the Department of Civil Registration in 5-year age groups.

Demographic data were classified according to the age groups of the registered TB cases.

We used the Disease Modeling (Dismode) software version II to analyze the data. Then, the World Health Organization Template was used to calculate the burden indicators (YLL and YLD).

DALYs presented in this study are age-weighted $(\beta=0.04)$ and are discounted for time preference $(r=0.03)$. For calculating the YLL, life expectancy is considered 82.5 years for females and 80 years for males $(5,6)$ based on the YLL software standards. In this study, the diagnosis of tuberculosis made with a definition that was used in the TB surveillance system by the ministry of health (4).

\section{Results}

In this study, 10661 individual data were analyzed. Of this, 5338 were male and 5323 were female.

The incidence and mortality in each age group are demonstrated in Tables 1 and 2. It shows that the incidence was higher in younger age groups.

The trend of incidence per 100.000 during a period of 12 years are depicted in Figure 1.

This trend was declining up to the year 2006 and then rose up to the year 2012, slightly. After 2005 we see an increasing trend in both genders and in 2012 this figure becomes closer in both male and female populations.

The YLL and YLD trend according to gender is demonstrated in Figure 2. The YLL is higher in men which suggests that life lost is higher in men. The YLD in men and women are very close.

Table 1. Incidence in each age group, according to gender

\begin{tabular}{lcccccccccccccccc}
\hline $\begin{array}{l}\text { Age } \\
\text { group }\end{array}$ & $\begin{array}{c}\text { Gender/ } \\
\text { Year }\end{array}$ & 2001 & 2002 & 2003 & 2004 & 2005 & 2006 & 2007 & 2008 & 2009 & 2010 & 2011 & 2012 & Total \\
\hline $0-14$ & Male & 247 & 335 & 226 & 146 & 143 & 104 & 128 & 128 & 144 & 148 & 168 & 220 & 2137 \\
& Female & 325 & 341 & 307 & 230 & 203 & 173 & 175 & 163 & 224 & 191 & 190 & 202 & 2724 \\
$15-$ & Male & 972 & 836 & 763 & 746 & 633 & 639 & 540 & 573 & 572 & 574 & 590 & 549 & 7987 \\
24 & Female & 1210 & 1045 & 921 & 846 & 758 & 771 & 818 & 803 & 849 & 818 & 870 & 791 & 10500 \\
$25-$ & Male & 932 & 926 & 908 & 998 & 863 & 881 & 858 & 852 & 903 & 892 & 1031 & 1073 & 11117 \\
34 & Female & 908 & 789 & 749 & 703 & 566 & 554 & 627 & 642 & 695 & 786 & 846 & 832 & 8697 \\
$35-$ & Male & 712 & 757 & 704 & 660 & 653 & 663 & 604 & 639 & 641 & 713 & 726 & 853 & 8325 \\
44 & Female & 784 & 674 & 644 & 557 & 513 & 484 & 469 & 494 & 543 & 595 & 570 & 583 & 6910 \\
$45-$ & Male & 593 & 597 & 564 & 584 & 536 & 579 & 559 & 556 & 600 & 619 & 601 & 609 & 6997 \\
54 & Female & 793 & 773 & 730 & 619 & 605 & 607 & 590 & 631 & 643 & 631 & 700 & 607 & 7929 \\
$55-$ & Male & 612 & 569 & 569 & 511 & 489 & 463 & 502 & 549 & 583 & 587 & 651 & 623 & 6708 \\
64 & Female & 839 & 806 & 804 & 729 & 637 & 645 & 695 & 973 & 764 & 751 & 808 & 790 & 9241 \\
$>65$ & Male & 121 & 126 & 123 & 114 & 117 & 120 & 123 & 126 & 129 & 141 & 146 & 140 & 1526 \\
& Female & 122 & 131 & 125 & 119 & 112 & 131 & 132 & 145 & 147 & 164 & 156 & 101 & 1585 \\
Total & Male & 4189 & 4146 & 3857 & 3759 & 3434 & 3449 & 3314 & 3423 & 3572 & 3874 & 3913 & 4067 & 44997 \\
& Female & 4981 & 4559 & 4280 & 3803 & 3394 & 3365 & 3506 & 3851 & 3865 & 3936 & 4140 & 3906 & 47586 \\
\hline
\end{tabular}


M. Khajedaluee, et al.

Table 2. The Death number in each age group, according to gender

\begin{tabular}{lcccccccccccccccc}
\hline $\begin{array}{l}\text { Age } \\
\text { group }\end{array}$ & Gender/Age & 2001 & 2002 & 2003 & 2004 & 2005 & 2006 & 2007 & 2008 & 2009 & 2010 & 2011 & 2012 & Total \\
\hline $0-14$ & Male & 7 & 7 & 8 & 5 & 5 & 2 & 9 & 5 & 5 & 6 & 10 & 11 & 80 \\
& Female & 10 & 10 & 9 & 7 & 6 & 3 & 4 & 5 & 12 & 5 & 10 & 9 & 90 \\
$15-24$ & Male & 29 & 25 & 23 & 22 & 18 & 12 & 16 & 20 & 11 & 11 & 11 & 8 & 206 \\
& Female & 12 & 11 & 9 & 8 & 9 & 7 & 12 & 2 & 13 & 12 & 14 & 5 & 114 \\
$25-34$ & Male & 47 & 46 & 45 & 50 & 6 & 35 & 54 & 41 & 44 & 60 & 63 & 46 & 537 \\
& Female & 9 & 8 & 7 & 7 & 6 & 15 & 9 & 6 & 10 & 11 & 12 & 11 & 111 \\
$35-44$ & Male & 46 & 49 & 46 & 43 & 42 & 44 & 55 & 39 & 52 & 50 & 55 & 49 & 570 \\
& Female & 16 & 14 & 13 & 11 & 10 & 12 & 12 & 15 & 11 & 12 & 15 \\
$45-54$ & Male & 47 & 48 & 45 & 46 & 45 & 49 & 40 & 32 & 49 & 50 & 54 & 36 & 541 \\
& Female & 24 & 23 & 22 & 19 & 18 & 21 & 21 & 32 & 28 & 22 & 32 & 29 & 291 \\
$55-64$ & Male & 61 & 57 & 57 & 51 & 48 & 46 & 51 & 55 & 43 & 55 & 70 & 53 & 647 \\
& Female & 42 & 40 & 40 & 36 & 33 & 34 & 36 & 36 & 41 & 37 & 36 \\
$>65$ & Male & 212 & 220 & 215 & 200 & 205 & 200 & 232 & 211 & 207 & 229 & 287 & 247 & 260 \\
& Female & 147 & 158 & 151 & 144 & 135 & 151 & 161 & 185 & 190 & 170 & 190 & 169 & 1951 \\
Total & Male & 449 & 452 & 439 & 417 & 369 & 388 & 457 & 403 & 411 & 461 & 550 & 450 & 5246 \\
& Female & 260 & 264 & 251 & 232 & 217 & 243 & 255 & 281 & 305 & 269 & 309 & 276 & 3162 \\
\hline
\end{tabular}

At the final step, we calculate the YLL, YLD and DALY trends in this population within 12 years (Fig. 3). It seems that YLL contributes more to the DALY, and there is a peak in the years 2007 and 2011.
Comparing the DALY in two genders, the DALY of TB was higher in men than women; and in men, there is the same peak in the years 2007 and 2011, but we can see different patterns in the female (Fig. 4).

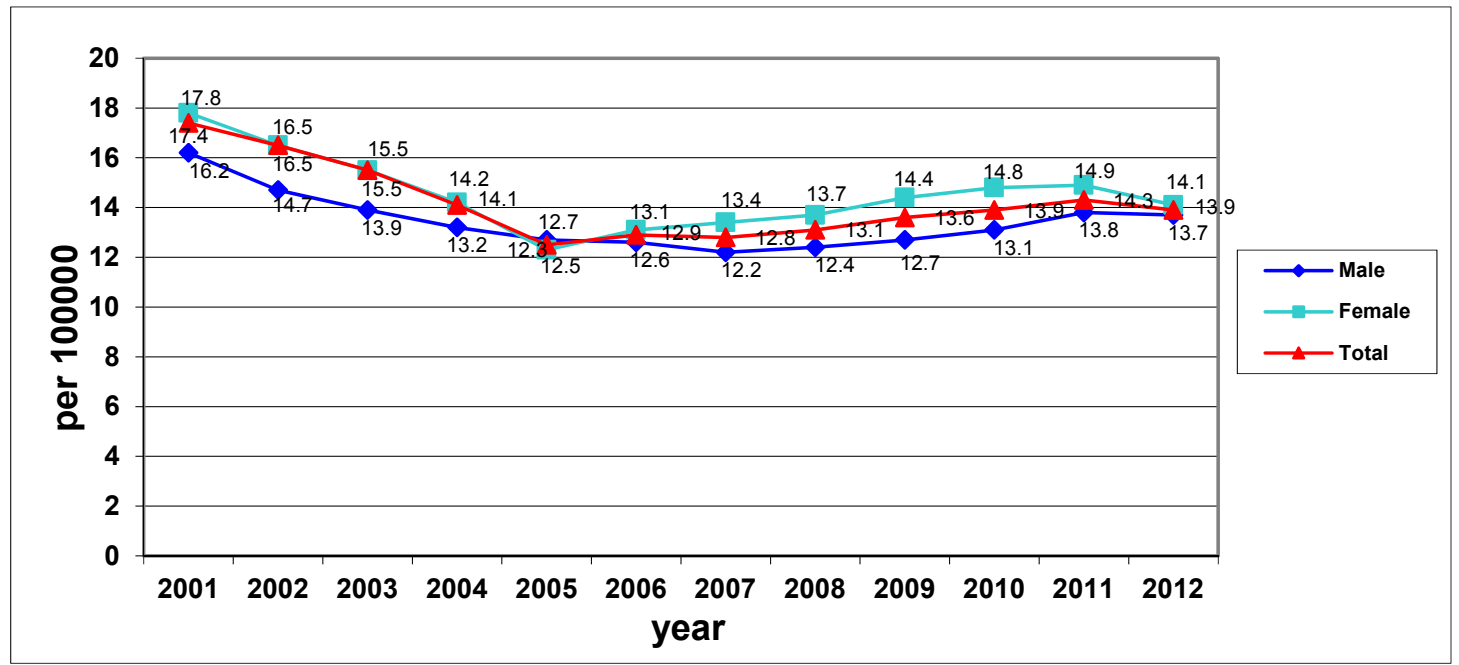

Fig. 1. The trend of incidence per 100.000 during 12 years, according to gender

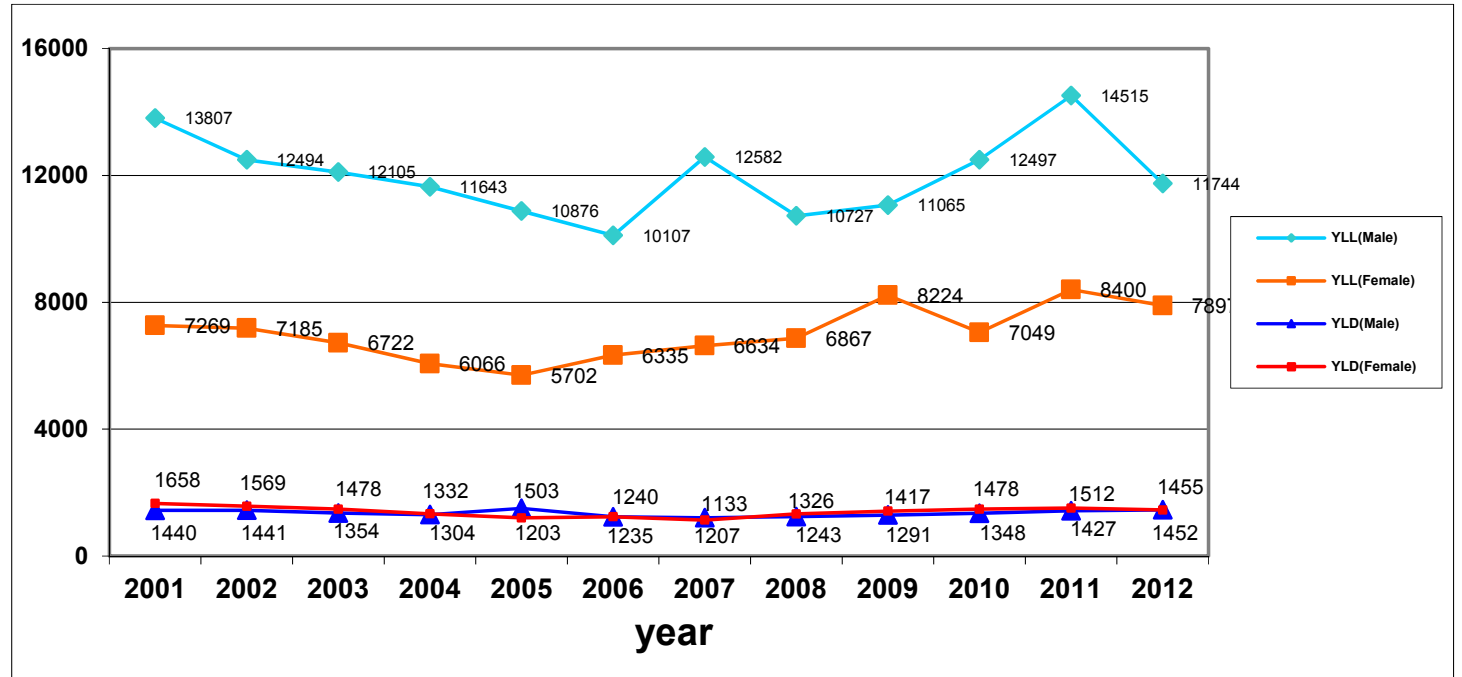

Fig. 2. YLL and YLD according to gender 


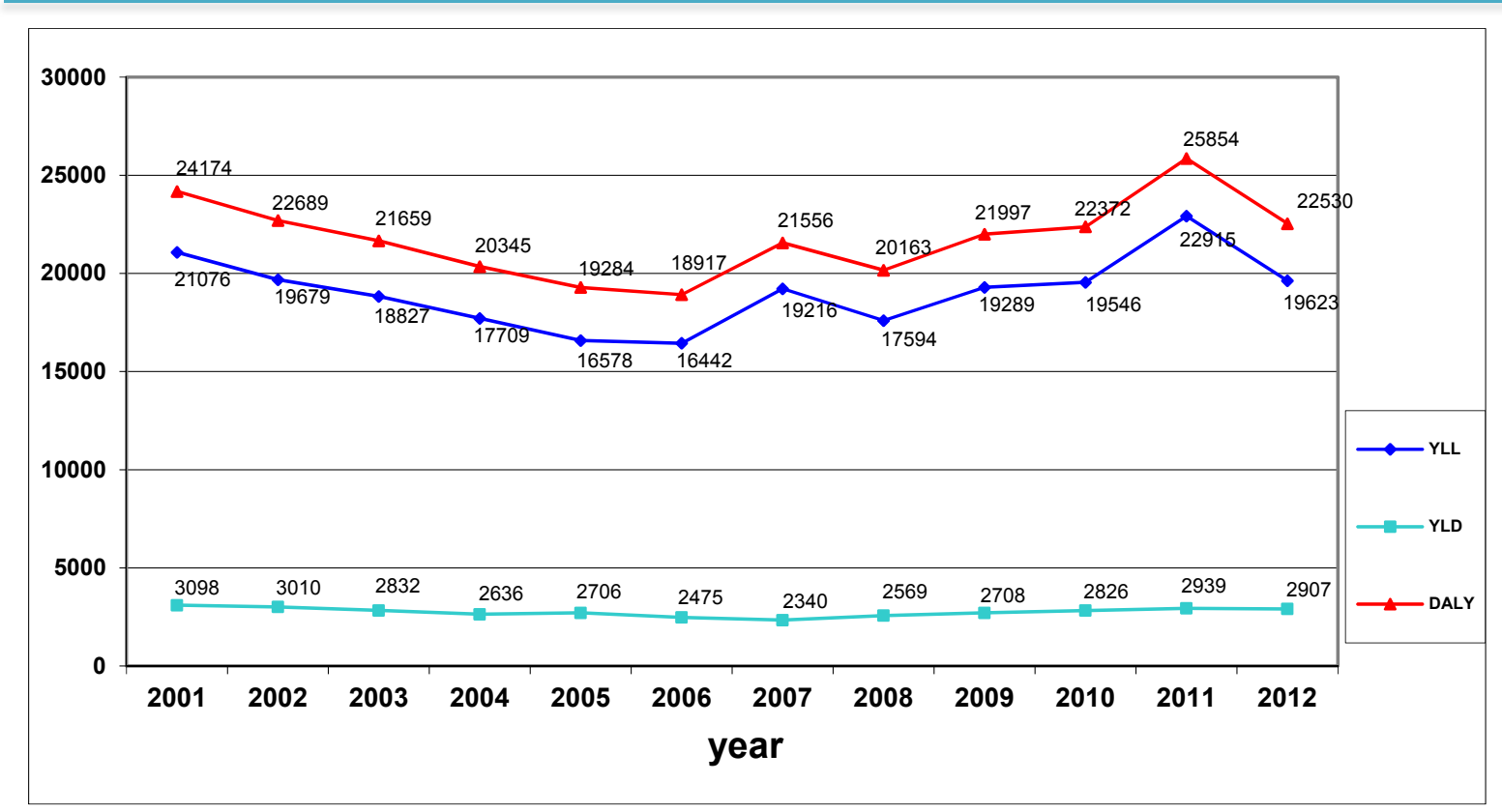

Fig. 3. The YLL, YLD, and DALY of TB in the population in 12 years

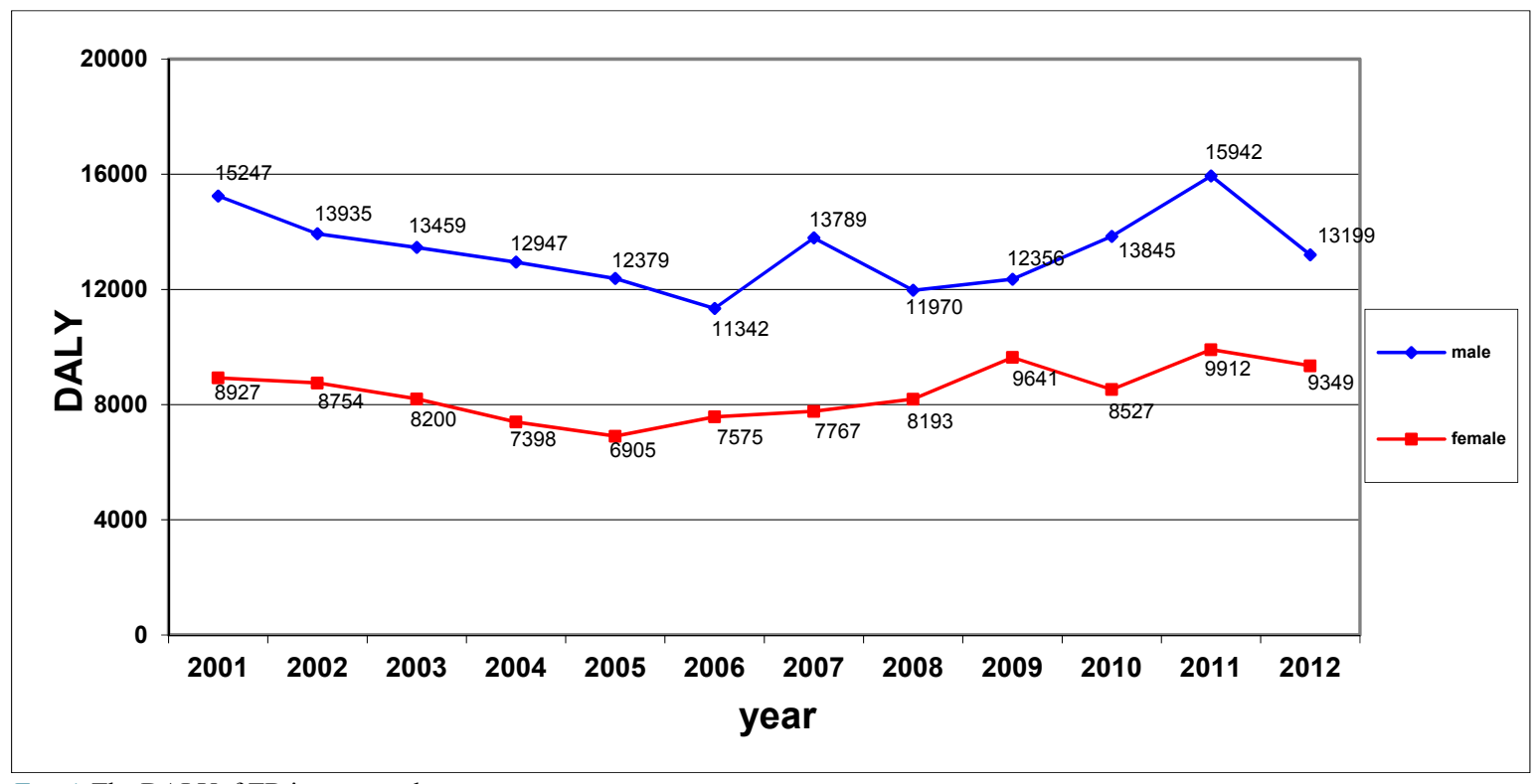

Fig. 4. The DALY of TB in two genders

\section{Discussion}

This 12-year population-based study in Iran, shows that the incidence rate from the year 2006 was changed from a decreasing trend to an increasing pattern in both gender and of the total population. The YLL was higher in the male population that led to higher DALY.

In this study, the mortality was higher in the elderly group that represents a significant success of the country in controlling the disease in younger age groups. Some studies suggest that TB is more difficult to diagnose in women, especially in developing countries and proposed that women have less access to TB treatment and prevention services than men and are unlikely to undergo smear examination (7). Socioeconomic factors may be the cause of the gender differences in the use of TB diagnosis services. For example, women in some contexts have difficultly accessing TB services because of some reasons: "Male family members and spouses are unwilling to pay for the health services for a female family member, women's health may not be considered important or because TB in women is more stigmatized than in men". In some communities, a woman with diagnosed TB may be divorced by her husband or may be excluded from the community or local groups (7).

Gender, tobacco, and TB are interlinked issues. It was stated that one out of five TB deaths is related to tobacco use (8). Generally, men are more prone to use tobacco, so we can conclude that this is a larger contributor to the TB disease burden for men than for women (9).

Globally, TB cases among men outnumber those among women, but we see exception in some countries such as 
Iran, Afghanistan, and regions of Pakistan (10). However, the reasons for this exception are not clear, but the Afghanistan National Strategic Plan for Tuberculosis Control said that it might be related to early marriage and short intervals between pregnancies (11) that can be similar to the context of our country in the past decade.

There is no consensus on the impact of occupational gender differences on TB infection rates. Some studies have suggested that men's greater engagement in certain risk factors such as smoking, drinking alcohol, and criminalized activities, can be related to higher infection rates. These associations are particularly strong with specific occupations such as deep pit mining (12). Similarly, it was suggested that women's work as a nurse or caregiver of a diseased person or household contacts with TB in close family members constituted a risk factor (13). It was suggested that women who expose to biomass fuel smoke are more likely to develop TB (14). In another studies, there was no increased TB risk due to exposure to cooking fires (before the illness), smoking fish, or burning items to keep insects away (13).

There are multiple probable reasons why the age differs between male and female patients. In most cases, young married women between the ages of 18 and 35 are expected to take care of everyone in the household. If a relative has tuberculosis, direct infection from a family member is quite possible by close contact (15).

The decrease in tuberculosis mortality rates is a global trend. The WHO reports that the absolute number of cases has fallen since 2006, as has its incidence since 2002 (16). This trend is almost observed in this study until 2006.

One reason for this decreasing trend since 1999 was the implementation of the directly observed treatment shortcourse (DOTS) in the world and in Iran.

Another reason may be the free and universal access to treatment and improvements in primary health care, especially the excellent primary health care in Iran.

In developing countries, it is estimated that $80 \%$ of the infected individuals are between 15 and 59 years of age (similar to this study) and are mostly men that are in the economically active age group that could have a negative impact on socio-economic growth (16).

However, the reference of the data used in this study is the official report of the national tuberculosis care system that is documented; our figures may differ from other studies that were done in Iran. It should be noted that the disease burden in this study was calculated based on the number of reported and registered tuberculosis cases in the national care system.

Neighbor countries of Iran (Afghanistan and Pakistan) which are among the 22 countries of the world's High Burden for TB, as well as Iraq (with its recent years of crisis) and the newly independent states of the north (with a high prevalence of multi-drug resistant tuberculosis), clearly shows that the need for attention to this disease is more than before.

\section{Conclusion}

In conclusion, although we should interpret these results with caution, but the use of DALY is recommended as a helpful tool to assess priorities. In the present investigation, it has been found that the overall tendency to get TB was higher in the female population. It shows that the incidence was more in younger age groups but the mortality was higher in the elderly group that shows the significant success of the country in controlling the disease.

\section{Acknowledgment}

Special thanks to Shahnaz Ahmadi and Hossein Samiei, who assist us in data collection, and the Ministry of health.

\section{Conflict of Interests}

The authors declare that they have no competing interests.

\section{References}

1. World Health Organization (WHO). (2011). Global Tuberculosis Control, http://www.who.int/tb/publications/global_report/2011/ gtbr11_full.pdf

2. Millennium Development Goals (MDGs). http://www.who.int/topics/ millennium_development_goals/en/

3. The ministary of health, communicable disease control center, Available at URL: http://tb-lep.behdasht.gov.ir/TB_Situation_in_Iran. aspx

4. The ministary of health, communicable disease control center, Available at URL:http://tb-lep.behdasht.gov.ir/Tutorial_Documents_ Inside.aspx

5. Khajedaluee M, Dadgarmoghaddam M, Saeedi R, Izadi-Mood Z, Abrishami M. The Burden of Diabetes in a Developing Country. Open J Prev Med. 2014:4:175-181.

6. Khajedaluee M, Dadgarmoghaddam M, Saeedi R, Izadi-Mood Z, Abrishami M, Zamani M. Mortality, Morbidity, Survival, and Burden of Top 9 Cancers in a Developing Country. Razavi Int J Med. 2014 August;2(3):e20073.

7. UNDP Discussion Paper: Gender and TB, December 2015 (Making the investment case for programming that addresses the specific vulnerabilities and needs of both males and females who are affected by or at risk of tuberculosis)

8. NCD Alliance, 'Putting noncommunicable diseases on the global agenda: NCD Alliance briefing paper: NCDs, Tobacco control, and the FCTC', NCD Alliance, 2011

9. Lim SS, Vos T, Flaxman AD, Danaei G, Shibuya K, Adair-Rohani H, et al. A comparative risk assessment of burden of disease and injury attributable to 67 risk factors and risk factor clusters in 21 regions, 1990-2010: a systematic analysis for the Global Burden of Disease Study 2010. Lancet. 2012;380(9859):2224-2260.

10. WHO, 'Tuberculosis in Women Factsheet 2014', WHO, Geneva, 2014, http://www.who.int/tb/publications/tb women factsheet 251013.pdf, cited in UNAIDS, 'Women Out Loud', UNĀIDS, Geneva, 2012.

11. Islamic Republic of Afghanistan, National Tuberculosis Control Program, 'National Strategic Plan for Tuberculosis Control 2014 2018', National Tuberculosis Control Program, Kabul 2013.

12. Argument referred to in: Mitchell, E.M.H., Gender trouble for Tuberculosis, Global Fund Observer Newsletter, Issue 254, 31 October 2014.

13. Crampin AC, Glynn JR, Floyd S, Malema SS, Mwinuka VK, Ngwira $\mathrm{BM}$, et al. Tuberculosis and gender: exploring the patterns in a case control study in Malawi. Int J Tuberc Lung Dis. 2004;8(2):194-203.

14. Narasimhan P, Wood J, Macintyre CR, Mathai D. Risk factors for tuberculosis. Pulm Med. 2013;2013:828939.

15. Chakraborty AK. Epidemiology of tuberculosis: Current status in India. Indian J Med Res. 2004 Oct;120(4):248-276.

16. Chirinos NEC, Meirelles BHS. Fatores associados ao ntegra do tratamento da tuberculose: Uma revisão ntegrative. Texto ContextoEnferm. 2011;20(3):599-406. 